\title{
Don't we need diagnostic facilities for genetically determined diseases?
}

\author{
SK Biswas
}

\author{
University of Tennessee Health Science Center, Memphis, Tennessee, USA
}

Most disease processes can be viewed as resulting from environmental influences interacting with the individual genetic makeup of the affected individual. A disease is genetically determined if it is mainly or exclusively caused by disorders in the genetic program of cells and tissues. More than 3000 defined human genetic diseases are known to be due to a mutation at a single gene locus (monogenic disease) and they follow a Mendelian pattern of inheritance. In addition, an important category of diseases results from genetic predisposition interacting with precipitating environmental factors and is known as multigenic or multifactorial diseases. This includes many relatively common chronic diseases such as hypertension, hyperlipidemia, diabetes mellitus, gout, psychiatric disorders and certain congenital malformations. Further categories of genetically determined diseases result from nonhereditary disorders in somatic cells (different forms of cancer) and chromosomal aberrations. Most cancers are now known to result from an accumulation of mutations in somatic cells.

Genetically determined diseases are not a marginal group but make up a substantial proportion of diseases. However, since genetic disorders affect all organ systems and age groups and are frequently not recognized, their contribution to the causes of human diseases appears smaller than it actually is. It is estimated that about $50 \%$ of spontaneous abortions during early months of gestation are due to a demonstrable chromosomal abnormality. About $1 \%$ of all newborn infants possess a gross chromosomal abnormality, and approximately $5 \%$ of individuals under the age of 25 years develop a serious disease with a significant genetic component. More than one-third of all pediatric hospital admissions are for diseases and developmental disorders that, at least in part, are caused by genetic factors. The lifetime frequency of genetic diseases is estimated to be 670 per 1000, including classic genetic disorders as well as cancer and cardiovascular diseases.

Everybody knows that the diagnosis and investigation of genetically determined diseases are similar to those of any other disease in that they require an accurate history, thorough clinical examination and appropriate use of diagnostic tests. However, it is increasingly evident that chromosomal and DNA analyses are not only important to confirm or exclude a diagnosis of genetic disease but also essential for many other settings. For example, chromosomal and genetic analyses are essential when there is a need of prenatal or pre-implantation diagnosis of a genetic disease and for identification of 'carriers' who do not suffer from the disease but carry the genetic abnormality. Genetic analyses also play important role in revealing the genetic make-up of family members of an affected person and thereby help providing appropriate genetic counseling. In addition, only genetic analyses can provide early diagnosis of those diseases that manifest only in adults and in older population. Furthermore, genetic analyses can be used for risk assessment for familial cancers especially those that originate in the hematopoietic system and for some other multifactorial diseases with significant genetic component.

In recent years, enormous progress of molecular biology and biotechnology and the success of human genome project have greatly influenced biomedical science. Many new disciplines like molecular medicine, molecular diagnostics and molecular and clinical genetics have evolved. The molecular genetics by utilizing molecular 

have largely improved the diagnosis as well as the management of genetically determined diseases. Gene therapy is not a dream any more and DNA/molecular genetic tests are routine investigations for a long list of genetic diseases and for both congenital and inherited cancer syndromes in many laboratories in developed countries. Such a list of diseases can be easily found in recent editions of any textbook of medicine including the Davidson's Principles and Practice of Medicine.

The diagnosis of a genetic disease is a multi-step procedure and it requires a systematic approach that takes clinical and genetic considerations into account. However, common techniques that are usually employed to investigate a genetic disease include karyotype analysis for identification of chromosomal structure and number, fluorescent in situ hybridization (FISH) for visualization of submicroscopic deletions, polymerase chain reaction (PCR) for detection of many common mutations, DNA sequencing for direct identification of mutations and microarray analysis to quickly identify small regions of DNA loss or gain.

Unfortunately, we have only a few or almost no diagnostic facilities for genetically determined diseases at the chromosomal or DNA level in Bangladesh. Patients with genetically determined diseases therefore suffer a lot. In most cases the diagnoses of genetic diseases are performed on the basis of clinical findings and findings obtained from traditional laboratory investigations. Physicians remain uncertain about the definitive diagnosis of patients suffering from genetically determined disease and therefore many of such patients do not get proper treatment and appropriate genetic counseling. The 'carriers' of genetic disease who are phenotypically normal and the family members of a genetically affected individual do not get any support and counseling from the physicians due to lack of facilities to identify the underlying genetic abormality in those cases. In some cases, samples are sent out to nearby 